\title{
Computer-aided destruction of complex structures by blasting
}

Steffen Mattern, Gunther Blankenhorn, Karl Schweizerhof Universität Karlsruhe, Institut für Mechanik

Institut für Mechanik

Kaiserstr. 12, Geb. 20.30

76128 Karlsruhe

Tel.: +49 (0) 721/ 608-2071

Fax: +49 (0) 721/ 608-7990

E-Mail: ifm@uni-karlsruhe.de

www.ifm.uni-karlsruhe.de 


\title{
Computer-aided destruction of complex structures by blasting
}

\author{
Steffen Mattern Gunther Blankenhorn \\ Karl Schweizerhof
}

\section{Introduction}

The controlled destruction of buildings at the end of their average life cycle has become more and more important during the last years. An economic way of dismounting is a demolition of a partially dismountable structure with explosives. Within this method, several structural elements of a building's load carrying system are removed by explosives to initiate a particular collapse. The strategy of blasting, i.e. the selection of the structural elements, which are destroyed by the explosives, is performed by engineers with special knowledge in this field of demolition. However the prediction of the collapse supported by fairly simple analysis models requires a great amount of experience and is very error sensitive if the building construction is complex.

In this contribution a 'complex construction' means a construction including several hundred structural elements like beams, plates, columns, etc. for which it is rather difficult to predict the collapse kinematics with simple mechanical tools. In order to develop a suitable strategy of blasting for complex structures, a reliable 'a-priori-simulation' of the collapse of the complete structure is mandatory. The goal of the investigations is to obtain a good prediction of the collapse mechanism by a numerical simulation of a complete real building. The analyses are performed using the Finite Element Method (FEM) combined with explicit time integration, which has already been approved as simulation tool for structural analyses involving impact and contact. Such rather costly simulations - costly with respect to the computational effort - are meant to support the development of a reliable and efficient simulation tool for demolition by blasting that will be developed throughout the research project FOR500 (funded by DFG). Complete FE-simulations of the failure process of a complex building require enormous computational resources and parallelization of such problems is inevitable. 
Within the contribution, first some general informations about the state of the art in building demolition is given. Then the methods used for the numerical analyses are discussed and the simulated reference models are presented. All computations and parametrical studies which were necessary to develop and improve the models were performed on the HP XC6000-Cluster of the University of Karlsruhe [2].

\section{Building demolition - State of the art}

Compared to methods such as destruction with dredgers or systematic deconstruction, blasting demolition definitely won recognition on braking down brickwork, reinforced concrete and steel constructions because of economic and technical reasons. Demolition by blasting, together with special possibilities of local weakening (e.g. cutting of reinforcement, deconstruction of supporting parts with special machines), allows the teardown of a building at a moderate time of preparation, proper planning of the blasting strategy and accurate positioning of the explosive charge presumed. Annoyance of residents is kept to a temporal minimum, business as usual and traffic around the object are disturbed only for a short while. The effort for necessary safety arrangements is considerably reduced compared to mechanical deconstruction, because the zone of danger can be evacuated during the collapse. For those reasons, popularity of blasting demolition raised in the last few years, especially in inner-city areas. General information about planning and accomplishment of a building demolition is given e.g. in [9].

Nevertheless, blasting demolition can be very dangerous, used for complex and non-trivial buildings, for which precise information about the construction details and the used materials is sometimes rare or even not available at all. The consequences can be recognized, when e.g. dynamic effects of the structure have not been considered correctly. This sometimes leads to uncontrolled collapses, which cause high damage. Dangerous situations can also arise, if a planned collapse is not completed and the remaining parts of the building have to be removed manually. In such situations expensive procedures are necessary to finish the demolition. Currently only several rudimental mechanical tools are used to predict the collapse, but in order to avoid accidents and the corresponding high costs, it is necessary to realize a reliable simulation of the complete collaps, which requires modern mechanical methods. 


\section{Numerical analysis}

\subsection{Basic idea}

The safe execution of the destruction of a building using controlled explosives requires detailed and reliable knowledge about the kinematics of the collapse with a special blasting strategy. In order to realize collapse simulations e.g. with different locations of the explosive loads, the Finite Element Method with explicit time integration is used within this project. Applying this method makes it possible to analyse the entire building from the time of blasting till the end of the breakdown. The knowledge won by those simulations is used to support and validate an alternative method based on rigid body models which requires far less computational effort. Such an efficient way of simulation allows the concerning of uncertainties e.g. with fuzzy algorithms [10], which require many deterministic solutions of one structure. The final goal here is to extract such models of reduced size based on certain modeling or decision rules by rigidizing parts of the structure during the simulation [1]. In order to gain proper insight and in particular, for validation purposes many analyses of different structures, i.e. extensive parametrical studies are necessary.

\subsection{Numerical algorithms}

First the numerical algorithms, used to solve this highly non-linear problem, have to be chosen. The problem is driven by finite deformations, finite rotations, non linear material behavior and multiple contact possibilities. On the other hand, the spatial discretization must be done by continuum elements to catch the contact surfaces in a better way, in comparison to structural elements. This leads to a large number of elements. Considering all requirements, a combination of $\mathrm{FE}$-analysis with explicit time integration $[3,8,5]$ is chosen. The small time step size, which is needed for numerical robustness, is compensated by highly efficient element formulations and the highly developed capabilities for dividing the problem to several computation units. Also the contact possibilities have to be taken into account which naturally reduce the possible time step size. As a conclusion the disadvantage of a small time step size in explicit methods - here the central difference scheme [11]- is abolished by its efficient implementation and parallelization [6]. 


\section{Reference Models}

\subsection{General Information}

In order to cary out the analyses, in this project the commercial FE-program LS-DYNA is used, which uses a central difference method for the time integration $[7,6]$. The code is highly parallelized and perfectly suited to run on clusters such as the HP XC6000. For discretization of the structural parts, one-point under-integrated hexahedral elements are chosen, which perform excellently without showing any locking. However a stabilization against unphysical element kinematics, the hourglass modes, is required. The chosen hourglass stabilization - Belytschko-Bindemann assumed strain co-rotational stiffness form, which performs extremely well - is described in detail in [4].

For all concrete parts, a piecewise linear plasticity material model is used. The parameters, necessary for concrete like behavior of this on purpose simplified material law were obtained by calibrating with rather simple experimental examples. However, though the material model does not allow detailed modification concerning e.g. reinforcement, the reached approximation for the investigated mass dominated problems was fairly good. The possibility of element failure, which is necessary to simulate the appearance of local zones of accumulated damage (hinges) during the collapse event is also implemented in the material model. E.g. every time an element reaches a specific plastic strain, it is removed from the computation. Especially this mechanism helps to support the development of rigid body models as mentioned in Section 3.1. Concerning accuracy, it is acceptable to use the same material model for each simulation since the spare available documentations of the objects contain little information about the used concrete and steel. Investigations with more detailed material models on local submodels of the simulated structure are carried out in another subproject involved in the Research Unit 500 [1].

Every time contact appears during the collapse event, the kinematical configuration of the simulated structure changes abruptly. Hence correct determination of contact within parts of the building and between building and ground is also very important to obtain realistic collapse behavior of a model. For this reason fast automatic contact search algorithms are implemented in LS-DYNA. Though these algorithms show very good performance concerning accuracy and computation time, the search for contact requires a considerable amount of CPU-time of the whole simulation as each surface segment of the FE-mesh has to be considered. The chosen contact formulation is a penalty based node-to-segment algorithm for building to baseplate contact and a segment-to-segment algorithm between the building parts in all inves- 
tigations. The base plate was modeled with four-node shell elements which were assumed to be rigid in each of the three presented models representing the contact segments for the base plate.

\subsection{Storehouse in Weida/ Thüringen}

As first example for a blast simulation, a storehouse in Thüringen was chosen, which has been demolished by blast in 1998. The framework of the seven storys was from reinforced concrete with masonry outer walls in the first and thin concrete walls in the upper floors. The building was $22 \mathrm{~m}$ high, $22 \mathrm{~m}$ long and $12 \mathrm{~m}$ wide with an approximate overall mass of 1900 tons. In the simulation, the collapse was reached by two steps of blasting as shown in Figure 1. With the first explosion, two rows of columns were removed, after four seconds, the third row was destroyed. The weakening, caused by the first explosion was not sufficient to start the collapse of the building, so it stayed for four seconds on the two remaining rows of columns. After the second explosion, the building started bending forward which lead finally to the collapse. First the complete upper six storys began to rotate, after the cuboid got into contact to the ground, it started to break into pieces.

The discretized structure as depicted in Figure 1, consists of 82867 hexahedral finite elements. Further informations about the simulation are given in Table 1. The results of a simulation can be seen in Figure 4.2. Unfortunately, the documentation of the real collapse of 1998 is limited to one movie, which makes the validation of the simulation difficult. However, the beginning of the collapse is fairly realistically captured, compared to the available data.

\subsection{Silo-building in Borna/ Sachsen}

The second reference model, computed on the HP-XC6000 Cluster is a silo building of about $25 \mathrm{~m}$ height with a base area of $36 \mathrm{mx} 12 \mathrm{~m}$ and an approximate mass of 3600 tons. All columns and girders, as well as the six collecting bins were from reinforced concrete. Also a few concrete walls were included for stiffening reasons, however, most of the outer walls were from masonry. Before the start of the blasting, the structure was weakened by removing wedge-shaped parts of the inner concrete walls as shown in Figure 3. The collapse in form of a bending movement to the front was realized by one explosion, removing the first row of columns completely. During the rotation and even after the first contact, the entire upper part stayed almost undeformed and was just turned over within the destruction. 


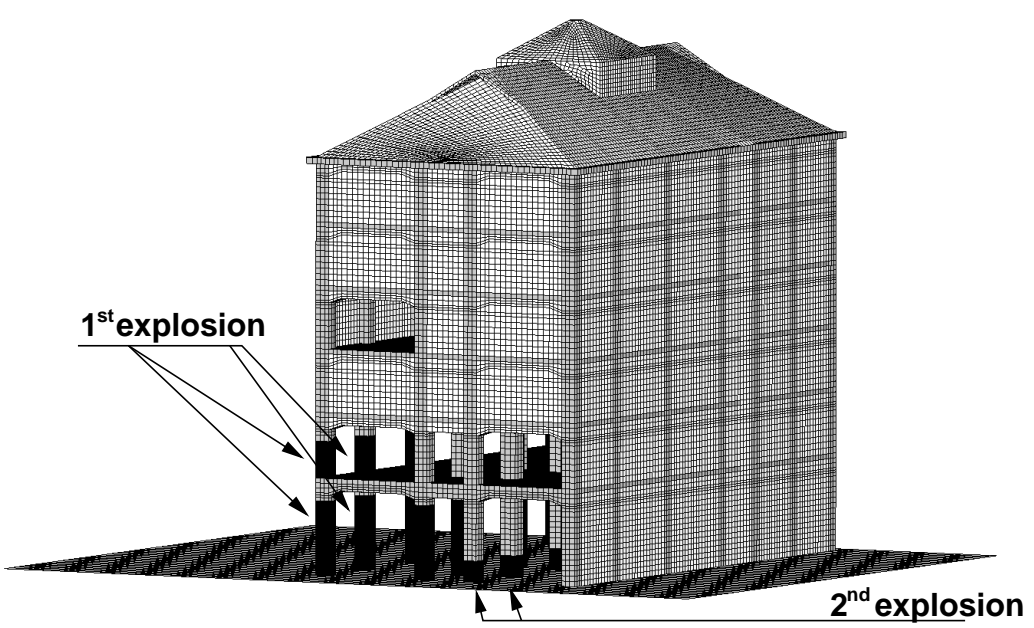

Figure 1: Finite Element model of storehouse in Weida/ Thüringen - Removal of columns by blast in two steps

\begin{tabular}{|l||c|c|c|}
\hline & Weida & Borna & Hagen \\
\hline \hline number of elements & 82867 & 77079 & 392481 \\
\hline simulated time & $9 \mathrm{~s}$ & $8 \mathrm{~s}$ & - \\
\hline number of processors & 8 & 8 & - \\
\hline total CPU-time & $65086 \mathrm{~s}$ & $124756 \mathrm{~s}$ & - \\
\hline CPU-time for contact search & $30 \%$ & $65 \%$ & - \\
\hline
\end{tabular}

Table 1: Comparison of the reference models - Weida (Section 4.2), Borna (Section 4.3) and Hagen (Section 4.4) 


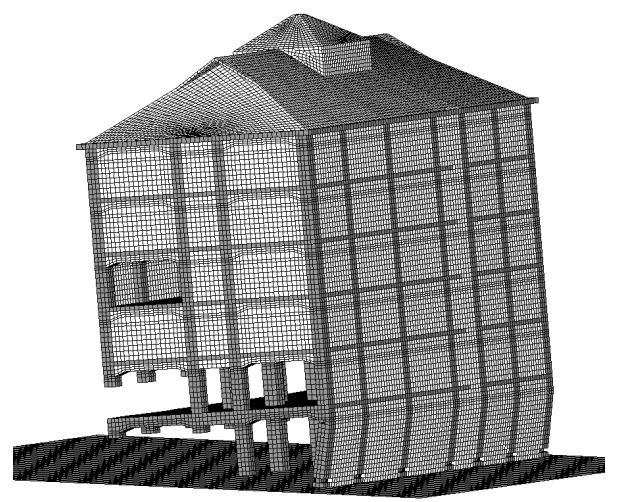

(a) $t_{1}=4.6 \mathrm{~s}$

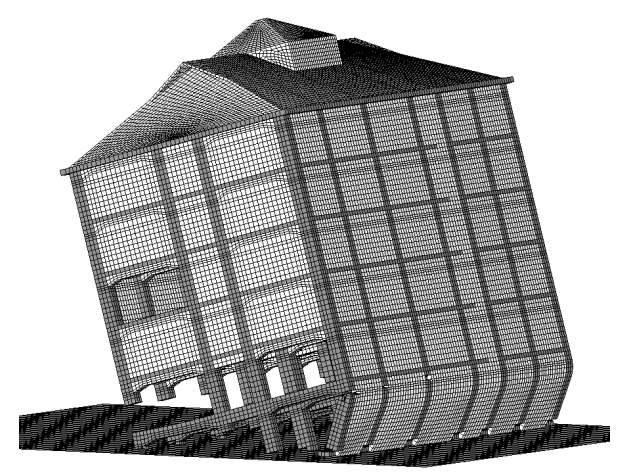

(b) $t_{1}=5.0 \mathrm{~s}$

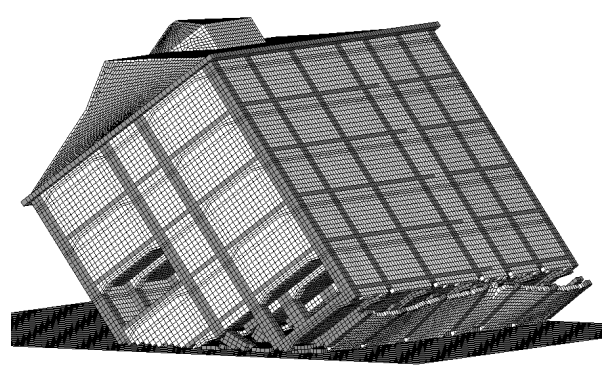

(c) $t_{1}=5.6 \mathrm{~s}$

Figure 2: Three States of the collapse simulation 


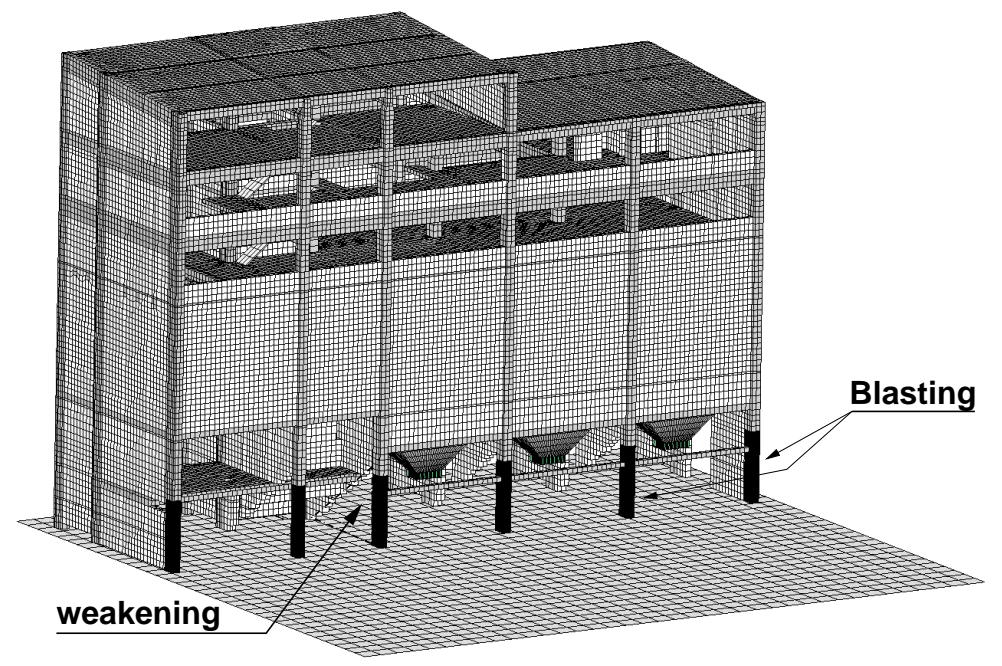

Figure 3: Finite Element model of silo-building in Borna/ Sachsen

The construction was discretized with 77079 hexahedral finite elements. As before, a rigid ground plate was modeled with shell elements, providing the segments for contact analysis. The discretized geometry is depicted in Figure 3. In Table 1 it can be seen, that the CPU-time needed for the a complete simulation is much higher, as for the simulation of the model from section 4.2 , though the number of elements and the simulated collapse time is smaller. The reason for this effect is the huge amount of CPU-time required for the contact search algorithm. The problematic part of this structure is at the funnels, were fairly small elements are necessary for discretization. After getting into contact with the ground plate, here the contact search algorithm needs a lot of time at every time step. In the future, alternative models have to be investigated in detail to achieve a more efficient analysis without sacrificing accuracy.

\subsection{Sparkasse in Hagen/ Nordrhein-Westfalen - High- rise with 22 storys}

The third and largest example, simulated within the project is a 22 store building in Hagen/ Nordrhein-Westfalen. The building with a mass of about 26500 tons is $93 \mathrm{~m}$ high, $37 \mathrm{~m}$ long and $19 \mathrm{~m}$ wide. The main part of the structure is a frame construction of reinforced concrete, stiffened against shear with concrete walls. The building had been broken down in 2004 . Before the blast process, all parts but the main load carrying construction 


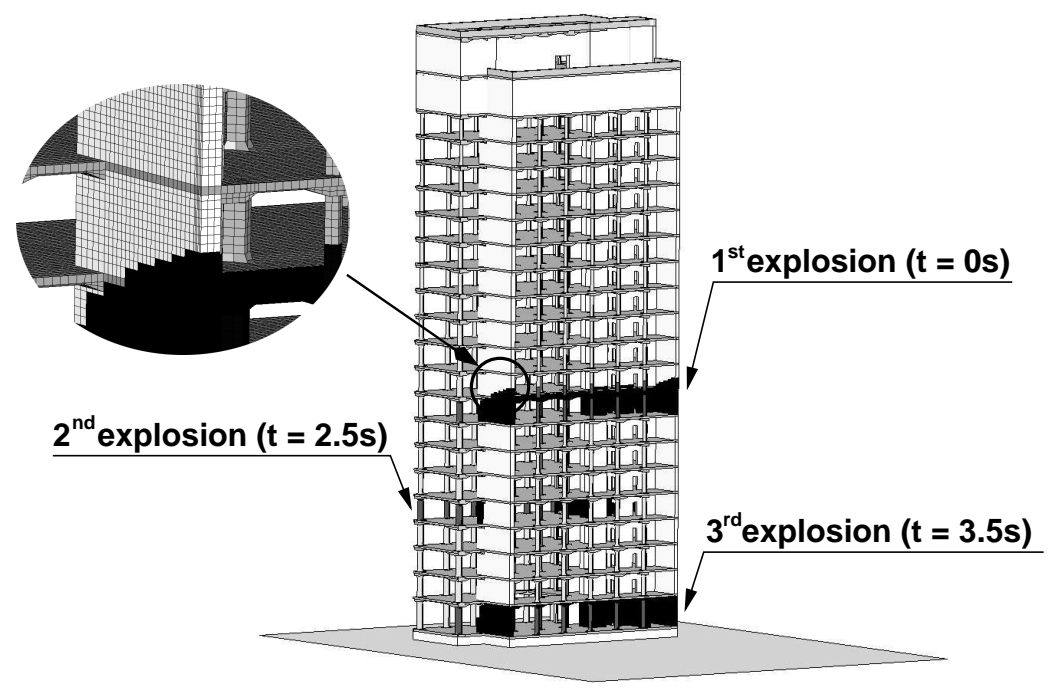

Figure 4: Finite Element model of the highrisebuilding in Hagen

were removed with machines or manually, also the facade was completely demounted. The planned collapse kinematic was reached by a combination of three explosions at $0 \mathrm{~s}, 2.5 \mathrm{~s}$ and $3.5 \mathrm{~s}$, where as shown in Figure 4 each time wedge-shaped parts were cut from the construction. The location of the weakened zones lead to a folding of the complete structure for reasons of minimizing of the space required for the debris.

In order to realize a rather regular mesh with fairly equal element sizes necessary for the proper simulation of the wave propagation process, 392481 solid elements were used for the discretization of the complete structure. Settings concerning contact or material were chosen with respect to the experience, gained by the simulation of the smaller buildings from sections 4.2 and 4.3. A complete simulation of the collapse of the building has not been carried out yet, so CPU-times are not known up to now. However the model is finished and the first complete run will be performed within the near future.

\section{Closure}

An insight into a part of the project work of the Reseach Unit 500 currently funded by DFG was given in this contribution. A number of fairly large Finite Element analyses have to be carried out in order to support the development of an alternative simulation process based on multi rigid body system analysis with comparable accuracy, but far less numerical effort. The development of the Finite Element models which is the focus of the current 
presentation itselves requires parametrical studies, to learn about e.g. contact or material parameters. These kinds of studies, especially with such large models are only possible, using high performance computers as the $H P$ XC6000-Cluster. However the project is not finished yet - as mentioned in Section 4.4 one model has not been simulated yet - but a lot of experience about the simulation of blasting demolition have already been reached during the last year. This knowledge is indispensable for the further investigations of the Research Unit 500.

\section{Acknowledgments}

The financial support of the German Research Foundation (DFG) (Project FOR 500 - Computer aided destruction of complex structures using controlled explosives) is greatfully acknowledged.

Providing every available data such as plans and video material is very important for the success of this project. For this support the authors like to thank Dr.-Ing. Rainer Melzer.

\section{References}

[1] DFG Research Unit 500. Computer aided destruction of complex structures using controlled explosives. http://www.sprengen.net/.

[2] Höchstleistungsrechner-Kompetenzzentrum Baden-Württemberg. http://www.hkz-bw.de/.

[3] K.-J. Bathe. Finite-Elemente-Methoden. Springer, 2002.

[4] T. Belytschko and L. P. Bindemann. Assumed strain stabilization of the eight node hexahedral element. Computer Methods in Applied Mechanical Engineering, 105:225-260, 1993.

[5] Ted Belytschko, Wing Kam Liu, and Brian Moran. Nonlinear finite elements for continua and structures. Wiley, 2000.

[6] J.O. Hallquist. LS-DYNA Theoretical Manual. Livermore Software Technology Corporation, 1991-1998.

[7] J.O. Hallquist. LS-DYNA Keyword User's Manual. Livermore Software Technology Corporation, 1992-2005.

[8] T. J. R. Hughes. The finite element method. Dover Publ., 2000. 
[9] J. Lippok and D. Ebeling. Bauwerkssprengungen. Weißensee-Verlag, 2006.

[10] B. Möller and M. Beer. Fuzzy randomness. Springer, 2004.

[11] W. L. Wood. Practical time-stepping schemes. Clarendon Pr., 1990. 